\title{
Investigation Protocol for Sensorineural Hearing Loss
}

\section{${ }^{1}$ Abir K Bhattacharyya, ${ }^{2}$ Jabin Thaj}

${ }^{1}$ Consultant ENT Surgeon and Royal College Surgical Tutor, Associate Director Medical Education (Surgery), Whipps Cross University Hospital, Whipps Cross Road, Leytonstone, London E11 1NR, United Kingdom

${ }^{2}$ Educational Fellow in Otolaryngology, Whipps Cross University Hospital, Leytonstone, London E11 1NR, United Kingdom

Correspondence: Abir K Bhattacharyya, Consultant Otolaryngologist and Head and Neck Surgeon, Battlehurst, 40 The Drive South Woodford, London E18 2BL, United Kingdom

\begin{abstract}
The etiology of sensorineural hearing loss is multifactorial which makes the diagnosis a challenging exercise and often an unsolved mystery. Whilst pure tone audiometry is a mandatory investigation, others such as laboratory and radiology are based on clinical evaluation. This paper puts forward a protocol for the investigation for sensorineural hearing loss.

Keywords: Sensorineural hearing loss, investigation, diagnosis, audiometry, otoacoustic emissions, evoked response audiometry, radiology, deafness, hearing loss.
\end{abstract}

\section{INTRODUCTION}

Sensorineural hearing loss (SNHL) refers to hearing impairment resulting from organic disorders of cochlea or the auditory nerve and its connections in brainstem. Normal hearing depends on integrity of the auditory pathway and on vascular, hematological, metabolic and endocrine systems. A variety of etiologies have been implicated (Table 1), and a wide range of investigations (Table 2) are available. However, pinpointing the diagnosis in sensorineural hearing loss can be a challenging exercise. In this paper, we attempt to put forward a protocol for the investigation of sensorineural hearing loss.

Table 1: Etiology

- Developmental and hereditary

- Nonsyndromic hereditary hearing loss

- Waardenburg's syndrome

- Alport's syndrome

- Usher's syndrome

- Inner ear anomalies

- Infectious disorders

- Labrynthitis

- Otitis media

- Viral infections

- Bacterial-syphilis

- Drug induced

- Renal

- Trauma

- Irradiation

- Neurological
Table 2: Investigations for sensorineural hearing loss

- Pure tone audiometry

- ECG

- Vestibular tests

- Radiographic tests

- Laboratory tests

- Other tests

- Speech audiometry

- Otoacoustic emissions

- Auditory brainstem response

\section{Audiometry}

Pure-tone audiometry (PTA) is a measure of threshold of hearing by air and bone conduction and helps to verify, classify and quantify hearing loss. It is the most simple and widely available technical investigation. Although it is a subjective measure, the test is very reliable if the patient is cooperative. ${ }^{1}$ Classification of hearing loss into conductive and sensorineural is further supported by tympanometry and acoustic reflex testing. Tympanometry is especially helpful in excluding the possibility of a conductive component in those with profound losses or bilateral losses in the presence of a masking dilemma.

PTA is the pivotal test that may help to differentiate between different types of SNHL (Flow Chart 1). SNHL can be symmetrical or asymmetrical based on the etiology. The common causes of asymmetric (unilateral) SNHL are sudden deafness, Ménière's disease and acoustic neuroma 


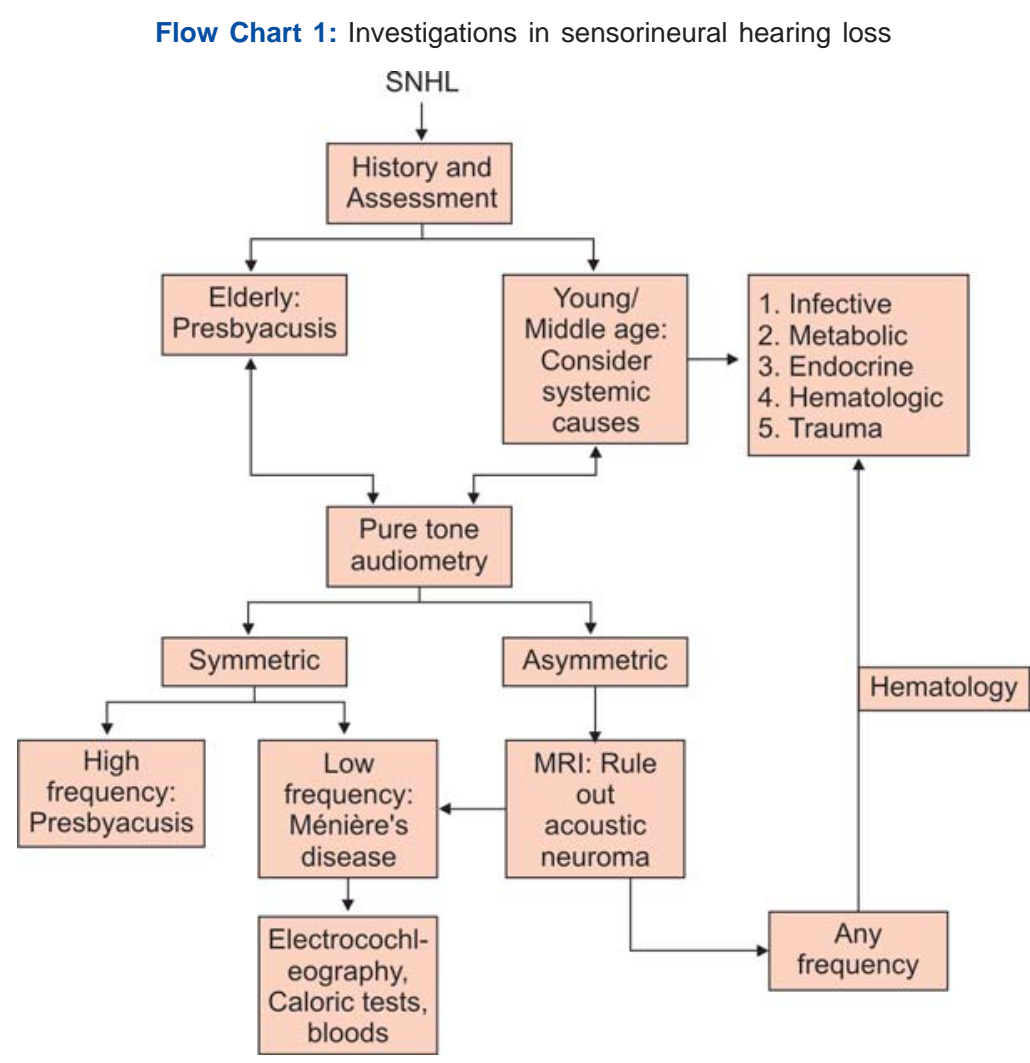

or other cerebellopontine angle tumors. ${ }^{2}$ Early diagnosis of retrocochlear pathologies is imperative and MRI is now the recommended investigation for this. ${ }^{2,3}$ Low frequency hearing loss is suggestive of Ménière's disease and this should be followed-up with supplementary investigations like vestibular function tests and electrocochleography. Atypical audiometric pattern in the young, with associated clinical features should prompt investigation into systemic causes of deafness like viral, bacterial, drug induced, autoimmune, radiation, trauma and neurological.

\section{Electrocochleography}

Electrocochleography (ECochG) measures electrical potentials arising in the cochlea and the auditory nerve within the first 5 milliseconds. The response is in the form of 3 phenomena: cochlear microphonics, summating potential SP and action potential AP of 8th nerve. SP and AP provide the most useful clinical information. Elevated SP: AP ratio is suggestive of Ménière's disease. ${ }^{4}$ ECochG is also useful in cases where wave 1 of $A B R$ is weak or absent. ${ }^{4,5}$ Other clinical applications of ECochG include intraoperative monitoring, cochlear implant candidates and stapedectomy. Studies have shown elevated AP/AP ratios in a series of patients with autoimmune disorders. ${ }^{6}$
There are 2 types of ECochG based on the position of the reference electrode: either on the promontory (transtympanic) or on the tympanic membrane (extratympanic). Both extratympanic and transtympanic electrodes have been found to be effective for ECochG evaluation. The extratympanic one causes less discomfort, but the transtympanic electrode produces tracings of better amplitude and reproducibility. ${ }^{7}$

\section{Vestibular Function Tests (Pertinent to SNHL)}

Vestibular function testing (VFT) is indicated in specific cases of SNHL. (Flow Chart 1). If the clinical features are suggestive of Ménière's (hearing loss, vertigo, tinnitus) and the audiogram shows characteristic pattern, VFT can be a useful adjunct. The most popular vestibular function test is electronystagmography, which helps in assessing the integrity of vestibulo-ocular and allied reflex systems. During attacks in patients with early Ménière's disease, the VOR gain toward the affected side is found to be higher than that toward the intact side, supporting the fact that the sensitivity of the cupuloendolymphatic system on the affected ear is increased. ${ }^{8}$ Amongst the various tests, the caloric test is 
the most informative. The percentage of canal paresis and directional preponderance can be calculated. Hypo activity of the affected side is seen in Ménière's disease. However, normal findings on ENG are not uncommon, especially if the test is done during disease free intervals.

\section{Radiology}

Patients with asymmetric hearing loss need further evaluation based on the degree of asymmetry. Various guidelines have been suggested for selection of patients for MR scanning to rule out acoustic neuroma. Using an interaural difference of $15 \mathrm{~dB}$ at 2 adjacent frequencies in unilateral hearing loss and $20 \mathrm{~dB}$ in bilateral hearing loss was found to have a sensitivity of $95 \%$ and specificity of $49 \% .^{9}$ A recent study has suggested a rule 3000 wherein asymmetry of $15 \mathrm{~dB}$ or more at $3000 \mathrm{kHz}$ requires an MR. If less than $15 \mathrm{~dB}$, a biannual audiometric follow-up is sufficient. ${ }^{10}$

MRI is found to be more sensitive than ABR in detection of small vestibular schwannomas. If MR is not available ABR is a suitable and sensitive investigation. ${ }^{11}$ Another study indicates that if waiting lists are long, the described strategy using ABR to select priority referrals for MRI scanning is recommended. ${ }^{12}$ Recent studies show that power spectrum auditory brainstem response (PSABR) could be a valid and reliable method of identifying subgroups of patients with unilateral auditory dysfunction who would best benefit from MRI. ${ }^{13}$

MRI scanning with gadolinium enhancement will identify virtually all tumors and was considered to be the gold standard. ${ }^{12}$ Recently T2-weighted fast spin echo MRI has been found to as sensitive as and less expensive than gadolinium enhanced standard MRI. ${ }^{14}$ All children with SNHL should undergo CT scan of the petrous bones/inner ear; abnormalities are more likely to be found in cases with craniofacial abnormalities, or profound or progressive hearing loss. The reported incidence of anomalies is 6.8 to $31 \%$ with dilated vestibular aqueduct being the commonest. ${ }^{15,16}$

Michel's aplasia, common cavity deformity and cochlear aplasia occurs due to arrest of development of inner ear structures at 3rd, 4th and 5th week of gestation respectively. Consequently, Michel's aplasia has the worst deformity with total absence of inner ear differentiation (Fig. 1). In common cavity deformity, the inner ear is a single cavity with no definite cochlea, vestibule and semicircular canals (Fig. 2).

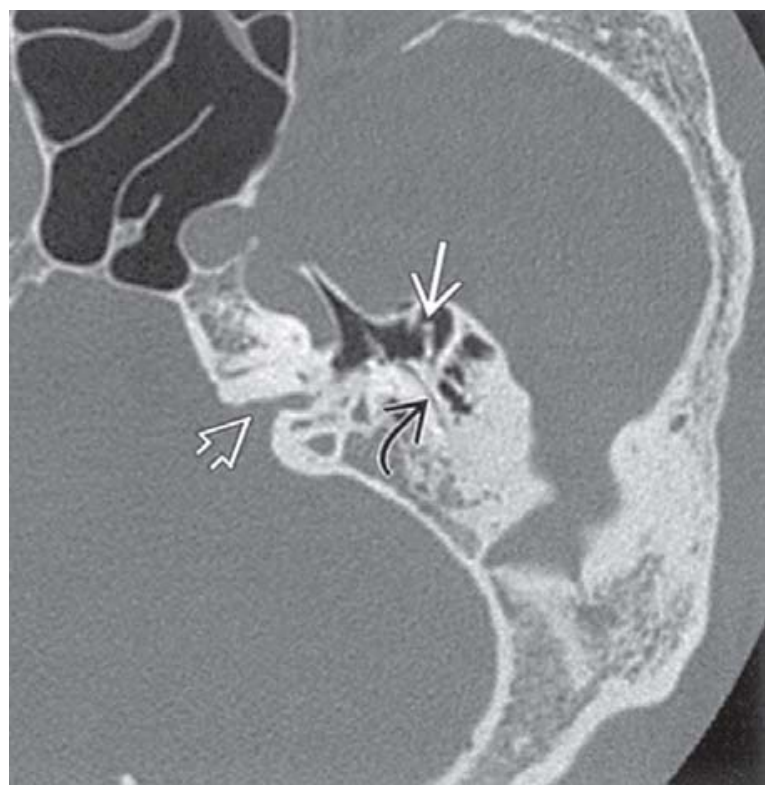

Fig. 1: Michel's aplasia. Aplasia of inner ear. CT showing no formation of inner ear structures with associated abnormalities. Curved arrow: abnormal course of facial nerve. Open arrow: Narrow IAC. Straight arrow: Abnormal ossicles
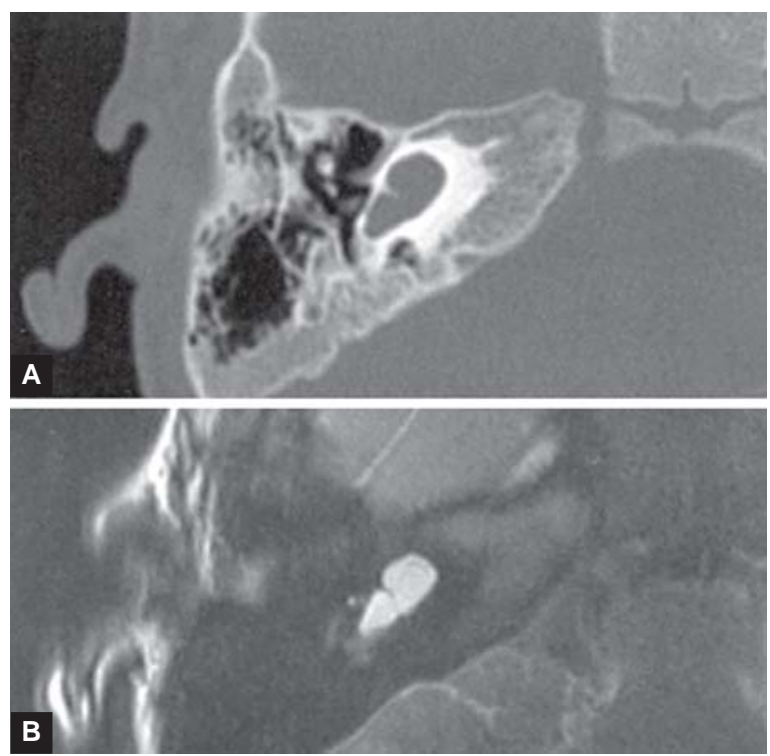

Figs 2A and B: Common cavity inner ear anomaly. CT (top) and MR (bottom). Cochlea and vestibule are a common communicating cavity without differentiation

In cochlear aplasia, cochlea is absent but vestibule and semicircular canals are present (Figs 3 and 4).

High-resolution multislice spiral computed tomography (MSCT) is especially suitable for the diagnosis of temporal bone fractures. ${ }^{17}$ The fracture line can be seen originating in occipital bone, extending anteriorly across the base of skull and petrous pyramid (Fig. 5). 

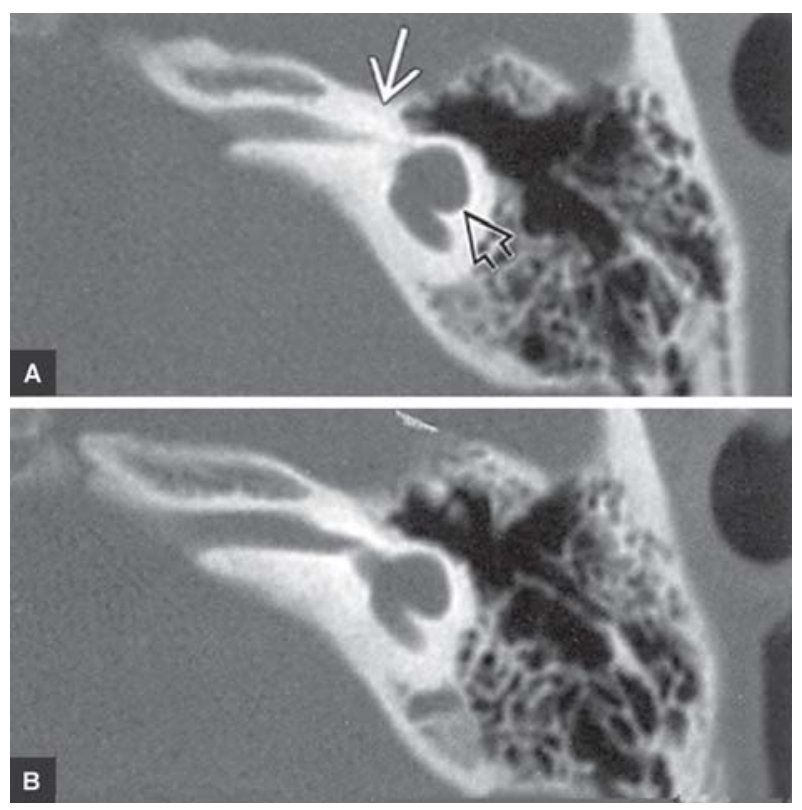

Figs 3 A and B: Cochlear aplasia. CT showing absent cochlea (arrow). Cystic vestibule (open arrow)
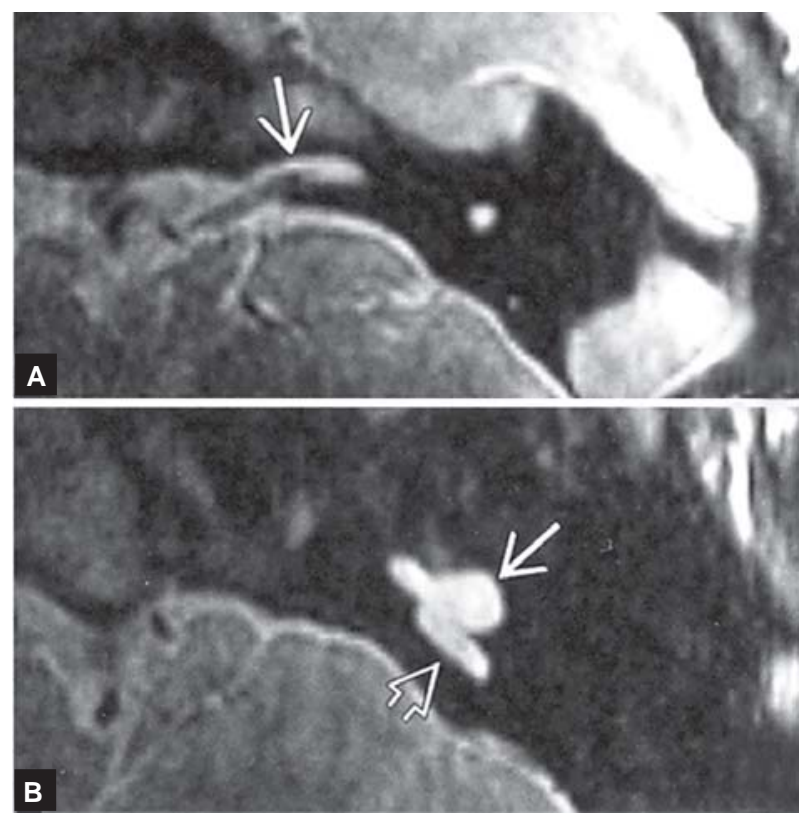

Figs 4A and B: Cochlear aplasia, MRI. Narrow IAC (arrow in A). Cystic vestibule (arrow in B). Dilated posterior semicircular canal (open arrow)

\section{Hematological investigations}

Systemic causes should be evaluated once presbyacusis, primary inner ear diseases like Mèniére’s, labrynthitis, etc. and retro cochlear causes of SNHL are excluded. The various medical causes of hearing loss should be kept in mind while assessing the patient. Basic hematological tests and biochemistry are more often than not, unhelpful. Syphilis though uncommon, is a potentially treatable cause of hearing

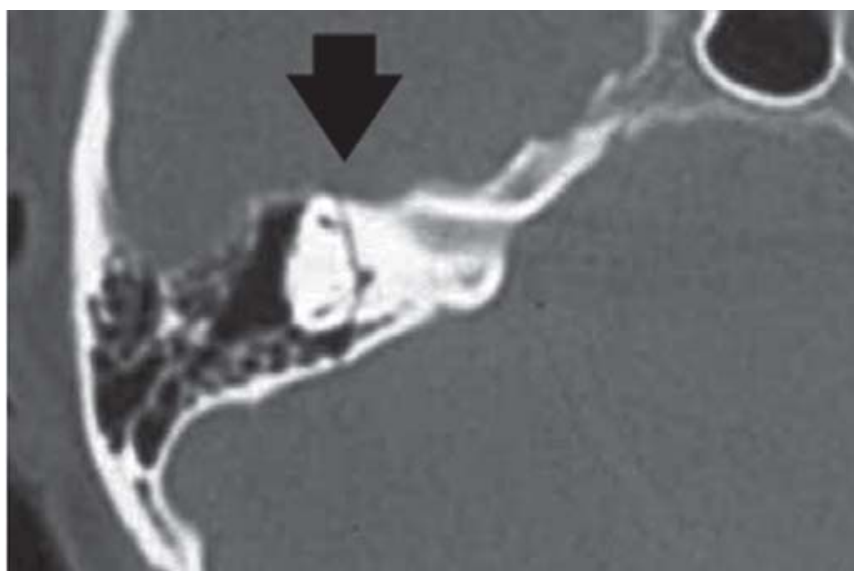

Fig. 5: Transverse fracture of temporal bone. CT showing fracture line running across the inner ear (bold arrow)

loss and deserves special attention. Fluorescent treponemal antibody test (FTA-ABS) and microhemagglutination test for syphilis are more sensitive than venereal disease research laboratory tests (VDRL).

Autoantibody testing in immune mediated SNHL is not sufficiently diagnostic with a study showing positive antinuclear antibody in only $34.4 \%$ of patients with autoimmune diseases. ${ }^{18}$ Immunobiological tests using fractioned human inner ear antigen have been used in the investigation of Mèniére's disease with equivocal results. ${ }^{19}$ Sensorineural hearing loss is common in children with congenital hypothyroidism and should be excluded by performing thyroid function tests. ${ }^{20}$ The reported incidence varies from 11.5 to $80 \% .{ }^{20,21}$ Studies have shown statistically significant improvement in hearing thresholds after treatment with levothyroxine. ${ }^{21}$

\section{Specific Audiometric Investigations}

A complete battery of supporting audiometric methods is available required for the differential diagnosis of different hearing disabilities including impedance, stapes reflex, speech audiometry, brainstem evoked response audiometry, otoacoustic emissions, speech discrimination tests, etc.

\section{Speech Audiometry}

Speech audiometry in conjunction with pure-tone audiometry can aid in determining the degree and type of hearing loss. This also provides information regarding discomfort or tolerance to speech stimuli and information on speech-discrimination abilities. 


\section{Otoacoustic Emissions}

Otoacoustic emissions (OAE) are acoustic signals detectable in the external auditory canal that reflect the state of health of the cochlea. Transiently-evoked otoacoustic emissions and distortion-project otoacoustic emissions are two types of otoacoustic emissions currently under investigation as well as in limited clinical use. ${ }^{22}$ These objective tests of cochlear function add significantly to the armamentarium currently in place for the evaluation of hearing. ${ }^{23}$

Otoacoustic emissions reflect the functional status of the outer hair cells and constitute the only noninvasive means of objective cochlear investigation. Transient evoked otoacoustic emissions are an extremely sensitive measure of the early effects of amino glycoside-induced injury to the peripheral auditory system. Therefore, their use is recommended for regular monitoring of cochlear function, in the presence of potentially toxic factors, aiming at prevention of permanent damage. ${ }^{24}$ The decreased DPOAE amplitudes at $4 \mathrm{kHz}$ in hypertriglyceridemic and diabetic patients without clinical findings are compatible with the sensorineural hearing loss observed with hyper viscosity and increased noise susceptibility. ${ }^{25}$

\section{Auditory Brainstem Response}

ABR is a noninvasive test that tests the auditory pathway from the eighth nerve to brainstem. It is a noninvasive test useful in assessment of hearing loss in newborn infants. ${ }^{26}$ ABR has been demonstrated to have low sensitivity and specificity in the evaluation of patients with retrocochlear pathology and cannot be relied on as a screening test for patients with asymmetric SNHL. Keeping the use of MRI conditional on the results of ABR will annually result in missed or delayed diagnosis of causative lesions in 29 patients per 1,000 screened. ${ }^{27} \mathrm{ABR}$ is under further evaluation to assess hearing loss in medical conditions. Multiple sclerosis is known to result in changes in wave morphology with increasing stimulation. ${ }^{28}$ Analysis of the latencies (waves I, II, III, and V) and the interpeak latencies (I-III and I-V) of BAEP revealed a significant difference between those of diabetics and those of healthy controls. The amplitudes of waves III, V, and I were definitely lower in comparison with amplitudes of healthy controls. ${ }^{29}$ Absent ABR waveforms in the presence of normal OAEs indicates an unusual type of hearing loss known as auditory dyssynchrony (auditory neuropathy). ${ }^{30}$

\section{REFERENCES}

1. Lemkens N, Vermeire K, Brokx JP, Fransen E, Van Camp G, Van De Heyning PH. Interpretation of pure-tone thresholds in sensorineural hearing loss (SNHL): A review of measurement variability and age-specific references. Acta Otorhinolaryngol Belg 2002;56(4):341-52.

2. Reiss M, Reiss G. Differential diagnosis of unilateral hearing loss. Praxis 2000 Feb 3;89 (6):241-47.

3. Mahillon V, Saussez S, Gérard JM, Chantrain G, Thill MP. Diagnostic management of unilateral sensorineural hearing loss in adults. Rev Med Brux 2003 Feb; 24(1):15-19.

4. Ruth RA, Lambert PR, Ferraro JA. Electrocochleography: Methods and clinical applications. Am J Otol 1998;9;1.

5. Lajtman Z, Borciæ V, Markov D, Popoviæ-Kovaciæ J, Vincelj J, Krpan D. Clinical interpretation of brainstem evoked response audiometry abnormalities in cochlear pathology. Acta Med Croatica 1999;53(3):119-23.

6. Kakigi A, Sawada S, Takeda T, Takeuchi S, Higashiyama K, Azuma H, Yamakawa K. Electrocochleographic findings in cases of autoimmune disease with sensorineural deafness. Auris Nasus Larynx 2003 Dec; 30(4):349-54.

7. Bonucci AS, Hyppolito MA. Comparison of the use of tympanic and extra tympanic electrodes for electrocochleography. Laryngoscope 2009 Mar; 119(3):563-66.

8. Maire R, van Melle G. Vestibulo-ocular reflex characteristics in patients with unilateral Ménière’s disease. Otol Neurotol 2008 Aug; 29(5):693-98.

9. Obholzer RJ, Rea PA, Harcourt JP. Magnetic resonance imaging screening for vestibular schwannoma: Analysis of published protocols. J Laryngol Otol 2004 May;118(5):329-32.

10. Saliba I, Martineau G, Chagnon M. Asymmetric hearing loss: Rule 3,000 for screening vestibular schwannoma. Otol Neurotol 2009 Jun; 30(4):515-21.

11. Baguley DM, Beynon GJ, Grey PL, Hardy DG, Moffat DA. Audiovestibular findings in meningioma of the cerebello-pontine angle: A retrospective review. J Laryngol Otol Nov 1997; 111(11):1022-26.

12. Ferguson MA, Smith PA, Lutman ME, Mason SM, Coles RR, Gibbin KP. Efficiency of tests used to screen for cerebellopontine angle tumours: A prospective study. Br J Audiol 1996 Jun; 30(3):159-76.

13. Dort JC, Cook EF, Watson C, Shaw G, Brown DK, Eggermont JJ. Power spectrum auditory brainstem response: Novel approach to the evaluation of patients with unilateral auditory symptoms. J Otolaryngol Head Neck Surg Feb 2009;38(1): 59-66.

14. Shelton C Harnseberger Allen R, King B. Fast spin echo magnetic resonance imaging: Clinical application in screening for acoustic neuroma. Otolaryngol Head Neck Surg 1996;114(1):71-76.

15. Hone SW, Smith RJ. Medical evaluation of pediatric hearing. Laboratory, radiographic and genetic testing. Otolaryngol Clin North America 2002;35:751-64.

16. Mafong DD, Shin EJ, Lalwani AK. Use of laboratory evaluation and radiologic imaging in the diagnostic evaluation of children with sensorineural hearing loss. Laryngoscope 2002;112:1-7.

17. Schuknecht B, Graetz K. Radiologic assessment of maxillofacial, mandibular, and skull base trauma. Eur Radiol 2005 Mar; 15(3):560-68 
18. García Berrocal JR, Ramírez-Camacho R, Vargas JA, Millan I. Does the serological testing really play a role in the diagnosis immune-mediated inner ear disease? Acta Otolaryngol 2002 Apr; 122(3):243-48.

19. Lejeune JM, Charachon R. New immunobiological tests in the investigation of Menière's disease and sensorineural hearing loss. Acta Otolaryngol 1992;112(2):174-79.

20. M Vanderschueren-Lodeweyckx, F Debruyne, L Dooms, E Eggermont, R Eeckels. Sensorineural hearing loss in sporadic congenital hypothyroidism. Arch Dis Child June 1983; 58(6):419-22.

21. Malik Vl, Shukla GK, Bhatia N. Hearing profile in hypothyroidism. Indian J of Otolaryngol Head Neck Surg April 2006; 233(44):312-14.

22. Rodriguez Jorge J, Zenner HP, Hemmert W, Burkhardt C, Gummer AW. Laser vibrometry. A middle ear and cochlear analyzer for noninvasive studies of middle and inner ear function disorders. HNO Dec 1997;45(12):997-1007.

23. Noel PE, Ramsey MJ, Amedee RG. Otoacoustic emissions: An emerging diagnostic tool. J La State Med Soc Apr 1995; 147(4):125-30.

24. Stavroulaki P, Apostolopoulos N, Dinopoulou D, Vossinakis I, Tsakanikos M, Douniadakis D. Otoacoustic emissions: An approach for monitoring aminoglycoside induced ototoxicity in children. Int J Pediatr Otorhinolaryngol Nov 5, 1999;50(3): 177-84.

25. Erdem T, Ozturan O, Miman MC, Ozturk C, Karatas E. Exploration of the early auditory effects of hyperlipoproteinemia and diabetes mellitus using otoacoustic emissions. Eur Arch Otorhinolaryngol Feb 2003;260(2):62-66.

26. American Academy of Pediatrics. Year 2000 position statement: Principles and guidelines for early hearing detection. Pediatrics 2000;106:798-817.

27. Cueva RA. Auditory brainstem response versus magnetic resonance imaging for the evaluation of asymmetric sensorineural hearing loss. Laryngoscope. Oct 2004;114(10): 1686-92.

28. Terk AR, Kveton JF.Clinical evaluation of hearing loss 174-82.

29. Tóth F, Várkonyi TT, Rovó L, Lengyel C, Légrády P, Jóri J, Czigner J, Kiss JG. Investigation of auditory brainstem function in diabetic patients. Int Tinnitus J 2003;9(2):84-86.

30. Berlin CI, Morlet T, Hood LJ. Auditory neuropathy/ dyssynchrony: Diagnosis and management. Pediatr Clin North Am 2003;331-40. 